\title{
Endoscopic combined middle and inferior meatal antrostomies approach in treatment of maxillary sinus inverting papilloma
}

\author{
Tomasz Gotlib, Magdalena Kuźmińska, Paulina Kołodziejczyk, Kazimierz Niemczyk \\ Department of Otolaryngology, Medical University of Warsaw, Warsaw, Poland
}

Videosurgery Miniinv 2020; 15 (4): 645-652

DOI: https://doi.org/10.5114/wiitm.2020.92309

\begin{abstract}
Introduction: Endoscopic medial maxillectomy is currently the most commonly used endoscopic approach to the maxillary sinus inverting papilloma. The possible complications of this procedure include epiphora, crusting and empty nose syndrome. Another method, a prelacrimal recess approach, enables preservation of the nasolacrimal duct and inferior nasal turbinate, but offers limited possibility of postoperative endoscopic follow-up.

Aim: To evaluate the combined middle and inferior meatus antrostomy approach to treat the maxillary sinus inverting papilloma.

Material and methods: A retrospective assessment of the medical records of consecutive patients operated on due to the maxillary sinus inverting papilloma in a single centre was performed.

Results: Fourteen patients operated on using combined antrostomies, with a follow-up of 2 years, are presented. In 2 patients, the tumor involved the prelacrimal recess. One of these patients was successfully operated on using combined antrostomies. In the second patient combined antrostomies were accompanied by a prelacrimal recess approach. None of the 14 patients had intraoperative complications. No recurrence was observed. No drying, empty nose syndrome, lacrimation, floppy turbinate or mucus recirculation was observed. Two patients complained of permanent cheek numbness. Follow-up endoscopy was easily performed with a rigid endoscope through both antrostomies in all patients except one.

Conclusions: Combined antrostomies appear effective to treat the maxillary sinus inverted papilloma. They are associated with minimal invasiveness and complications and enable easy endoscopic follow-up. In patients with prelacrimal recess involvement, it can be supplemented by the prelacrimal recess approach or, if needed, converted to an endoscopic Denker procedure.
\end{abstract}

Key words: maxillary sinus, inverted papilloma, endoscopic sinus surgery, inferior antrostomy, middle antrostomy.

\section{Introduction}

Inverted papilloma (IP) is a benign epithelial sinonasal tumor most commonly occurring in the maxillary sinus and the lateral wall of the nasal cavity. It has a potential for malignant transformation and a tendency for recurrence if incompletely removed. Multiple attachment is associated with increased risk of recurrence [1]. The majority of recurrences take place within 2 years. There is evidence suggesting that 5-10 years or even lifelong follow-up is needed, as $6 \%$ of IPs reoccur after 10 years [2]. Traditionally IP was removed using Denker's approach or medial maxillectomy (via lateral rhinotomy or midfacial degloving). Currently the most commonly used endoscopic approach to the maxillary sinus IP is endoscopic medial maxillectomy. The endoscopic approach results in a lower recurrence rate. This can

\footnotetext{
Address for correspondence

Paulina Kołodziejczyk MD, Department of Otolaryngology, Medical University of Warsaw, 1 a Banacha St, 02-097 Warsaw, Poland, e-mail: kolodziejczyk.paulina11.02@gmail.com
} 
be attributed to better surgical vision compared to traditional approaches.

The potential complications of endoscopic medial maxillectomy include epiphora, crusting and empty nose syndrome. There is a growing number of studies confirming that the prelacrimal recess approach, which enables preservation of the inferior turbinate and nasolacrimal duct, is equally effective $[3,4]$. Combined middle and inferior meatus antrostomies enable a good approach to the maxillary sinus including its anterior wall [5]. This approach can be insufficient in patients with prelacrimal recess involvement. However, it allows not only for preservation of the nasolacrimal duct and inferior nasal turbinate but also easy endoscopic follow-up. Currently available computed tomography (CT) and magnetic resonance imaging (MRI) imaging enables prediction of the site of tumor attachment and iden- tification of patients who can benefit from the combined antrostomies approach [6].

\section{Aim}

The aim of this study is to evaluate combined middle and inferior meatal antrostomy approach in treatment of maxillary sinus inverted papilloma.

\section{Material and methods}

Retrospective assessment of medical records of consecutive patients operated on due to maxillary sinus inverted papilloma operated by a single operator was performed.

Exclusion criteria: recurrence of inverted papilloma after previous procedures with inferior turbinate removal. The university ethics committee approved the study.
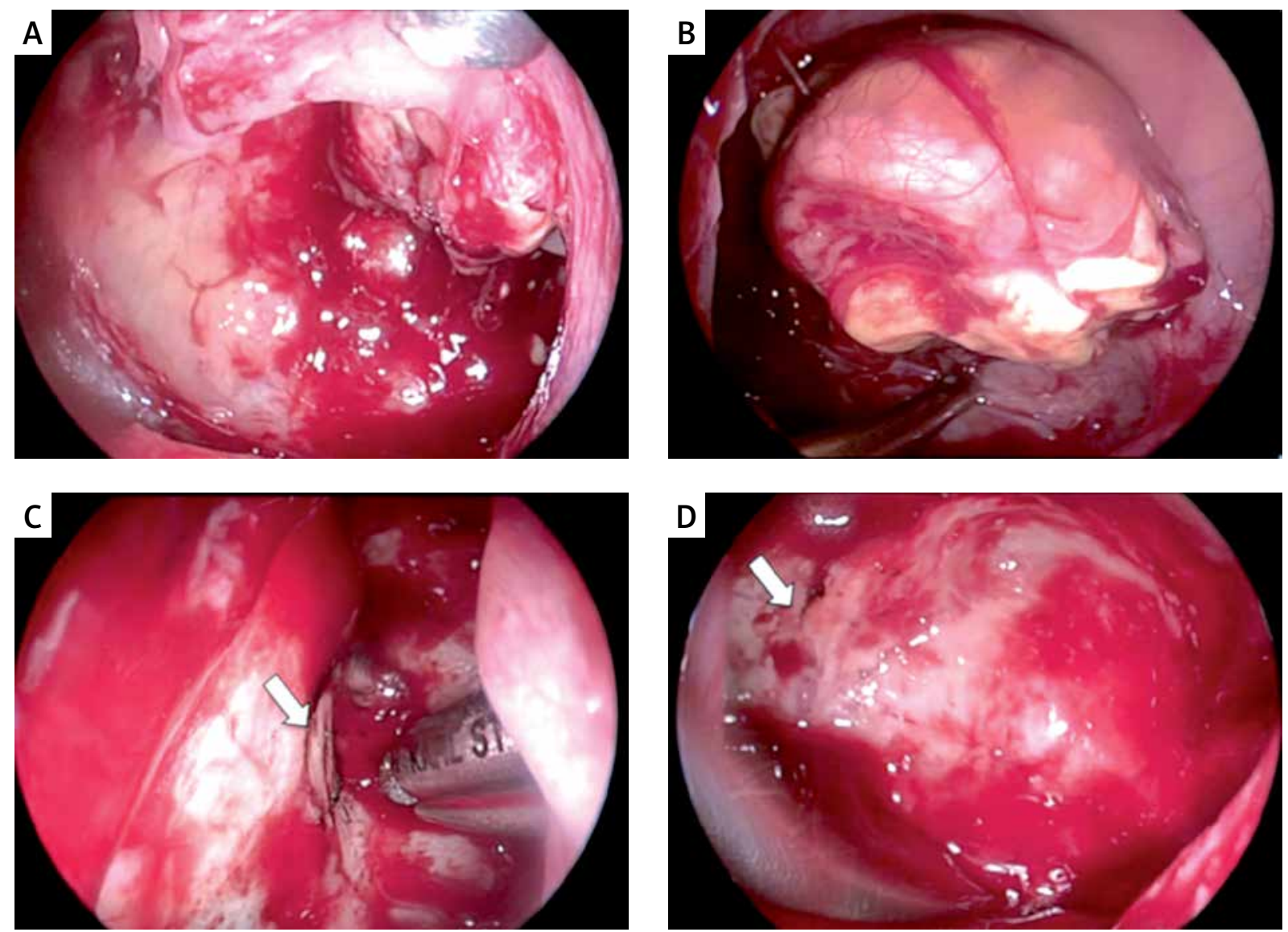

Photo 1. Intraoperative view of the left maxillary sinus. A - middle antrostomy view: the tumor is only partially visible, B - inferior antrostomy view: the tumor is much better exposed, C - removal of the tumor attachment: middle antrostomy view, the burr is introduced through the inferior antrostomy, D - the view through the inferior antrostomy at the end of the procedure. White arrow - infraorbital nerve 


\section{Surgical technique}

The surgical procedures were performed under general anesthesia. Pledgets soaked in adrenaline were applied in the nasal cavity, under the middle and inferior turbinate.

The axilla of the middle turbinate and the lateral nasal wall under the inferior turbinate were infused with 1\% lidocaine and 1:100 000 adrenaline solution. The intranasal part of the tumor was removed (if present), then uncinectomy and wide middle meatal antrostomy (MMA) were performed (Photo $1 \mathrm{~A}$ ). The free-floating part of the tumor was resected using angled forceps or a microdebrider. The maxillary sinus was inspected with 30,45 and $70^{\circ}$ rigid scopes or an adjusted viewing angle scope $\left(0-90^{\circ}\right)$. The inferior meatal antrostomy (IMA) was created typically $0.5 \mathrm{~cm}$ posterior to the anterior insertion of the inferior turbinate using the curette and cutting forceps. Care was taken to avoid stripping the mucosa and injuring Hasner's valve. In case of anterior wall involvement the inferior turbinate was gently fractured and displaced medially to gain space for the endoscope and surgical tool, then the IMA was widened anteriorly using backbiting forceps and an irrigated diamond burr to the edge of the pyriform aperture. Resection of the middle wall of the sinus was tailored to the disease. If needed, middle and inferior antrostomies were merged together leaving the inferior turbinate suspended between its anterior and posterior attachment. Depending on anatomical conditions and location of the tumor attachment both antrostomies were used for sinus inspection and/or insertion of the surgical tools (Photo 1). After identification of the tumor pedicle, the surrounding mucosa was coagulated and removed, and underlying bone was drilled down with a curved diamond burr (Photos 1 C, D). Postoperative care included saline douches and debridement under endoscopic guidance 2 weeks postoperatively, and further regular endoscopic follow-up.

\section{Results}

From 2010 to June 2017, a total of 14 patients meeting the study criteria underwent surgery for maxillary sinus IP performed by a single operator (T.G). There were 7 women and 7 men, with a mean age 58.5 years (range: $47-76$ years). Six of these patients were followed for 6 years or longer. Three patients were operated on due to IP recurrence including one after a previous Caldwell-Luc procedure and two after incomplete removal through the middle antrostomy (Table I). Preoperative imaging included $\mathrm{CT}$ and in 4 patients also MRI. Tumor attachment was identified on preoperative CT and/or MRI in 10 patients (Photos 2 and 3). Location of tumor pedicle and patient's data are presented in Table I.

Table I. Summary of patient data $(n=14)$

\begin{tabular}{|c|c|c|c|c|c|c|c|}
\hline $\begin{array}{l}\text { Patient } \\
\text { no. }\end{array}$ & $\begin{array}{l}\text { Pedicle location } \\
\text { at surgery }\end{array}$ & $\begin{array}{c}\text { Previous } \\
\text { surgery }\end{array}$ & Approach & $\begin{array}{c}\text { Preop. CT/ } \\
\text { MR }\end{array}$ & $\begin{array}{c}\text { Pedicle visibleon } \\
\text { CT or MR }\end{array}$ & $\begin{array}{l}\text { Postop. } \\
\text { imaging }\end{array}$ & $\begin{array}{c}\text { Follow-up } \\
\text { [years] }\end{array}$ \\
\hline 1 & LW & & CA & CT & Yes & (Endo) & 9.5 \\
\hline 2 & LW & & CA & $\mathrm{CT}$ & No & CT & 7.5 \\
\hline 3 & IC/SW & & CA & $\mathrm{CT}, \mathrm{MR}$ & Yes & CT & 7 \\
\hline 4 & IC/SW & & CA & $\mathrm{CT}, \mathrm{MR}$ & Yes & CT & 7 \\
\hline 5 & AW/MW/PR & $\mathrm{CL}$ & CA & CT & No & CT & 7 \\
\hline 6 & MW & & CA & CT & No & (Endo) & 7 \\
\hline 7 & MW & & CA & CT & No & MR & 4 \\
\hline 8 & AW/LW/SW & & CA & $\mathrm{CT}, \mathrm{MR}$ & Yes & $\mathrm{CT}, \mathrm{MR}$ & 3 \\
\hline 9 & AW & & CA & CT & Yes & CT & 2.5 \\
\hline 10 & IC & & CA & $\mathrm{CT}, \mathrm{MR}$ & Yes & CT & 2.5 \\
\hline 11 & AW/MW/PR & FESS & $C A+$ PreLA & CT & Yes & MR & 2.5 \\
\hline 12 & AW/LW & FESS & CA & CT & Yes & CT & 2 \\
\hline 13 & AW/LW & & CA & CT & Yes & CT & 2 \\
\hline 14 & LW & & CA & CT & Yes & $\mathrm{CT}, \mathrm{MR}$ & 2 \\
\hline
\end{tabular}

$L W$ - lateral wall, IC - infraorbital canal, SW - superior wall, AW - anterior wall, $M W$ - medial wall, PR - pre-lacrimal recess, CA - combined antrostomies, PreLA - pre-lacrimal approach, CL - Caldwell-Luc approach, (endo) - endoscopic follow-up. 

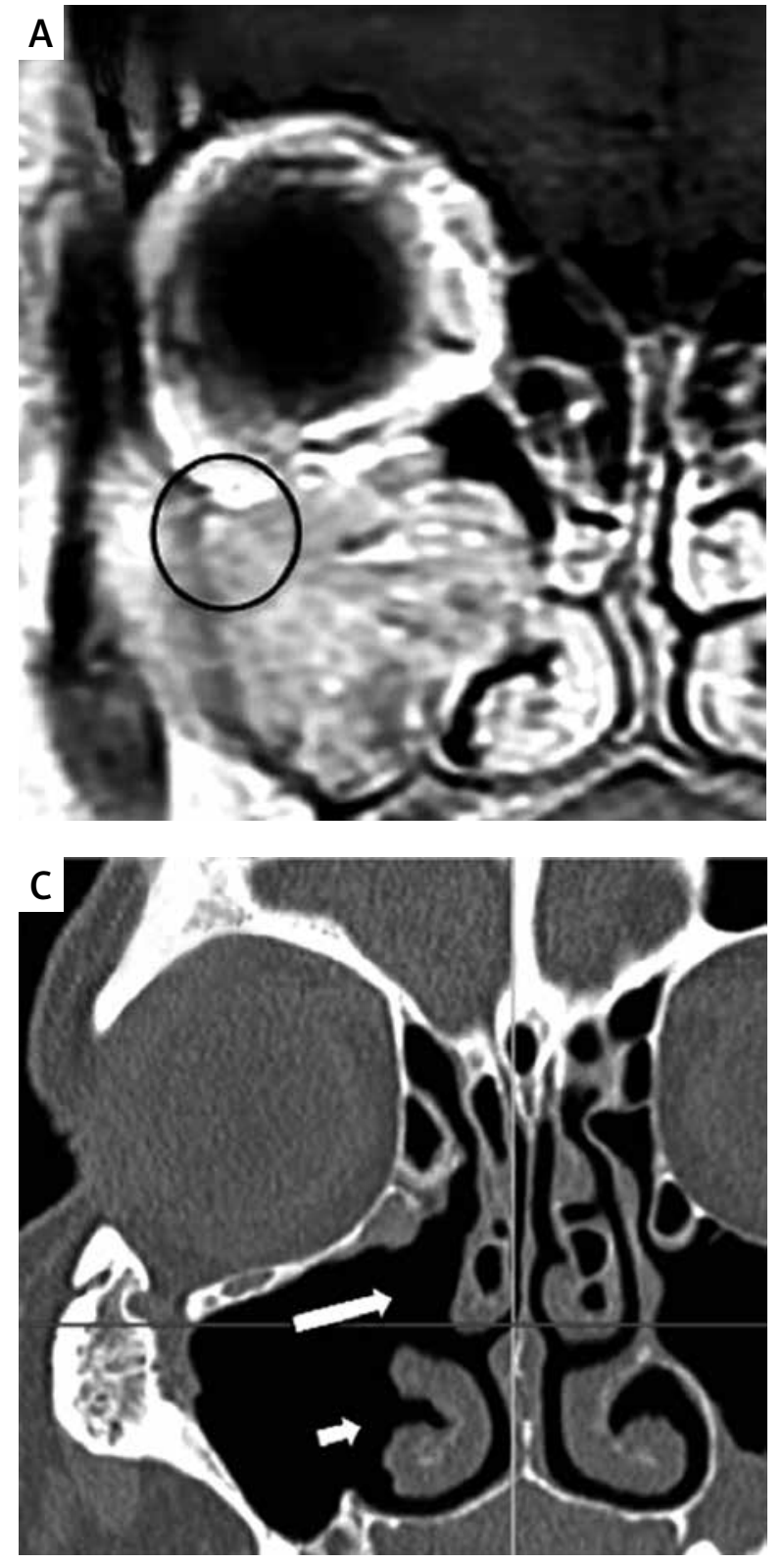

There were no cases with multifocal attachment or malignant transformation. In 1 of the 2 patients with prelacrimal recess involvement combined antrostomies allowed efficient control of the recess due to favorable anatomic conditions. In the second one combined antrostomies were supplemented by a prelacrimal approach.

Postoperative CT and/or MRI was performed in 11 patients. The three remaining patients were followed up with endoscopic examinations only. The postoperative follow-up was possible through IMA in

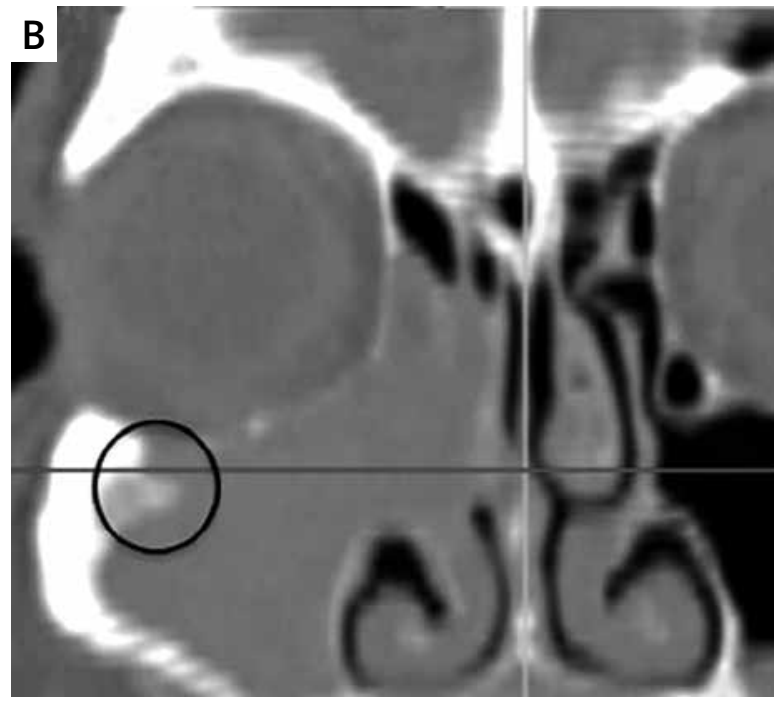

Photo 2. Preoperative $C T$ and $M R I(A, B)$ and postoperative $C T$ (C) scans of patient no 8: red arrow - tumor attachment, white arrow - inferior meatal antrostomy, blue arrow - middle antrostomy

13 patients with a rigid scope. In most cases, there was no need for local anesthesia or decongestion. In 1 patient synechiae formation narrowed the middle antrostomy, making endoscopic follow-up with a rigid scope difficult.

No lacrimation, drying, empty nose syndrome, floppy turbinate or mucus recirculation was observed. Two patients with an IP pedicle attached to the infraorbital canal complained of permanent cheek numbness, which could be the result of drilling or use of diathermy. No recurrence was observed. 


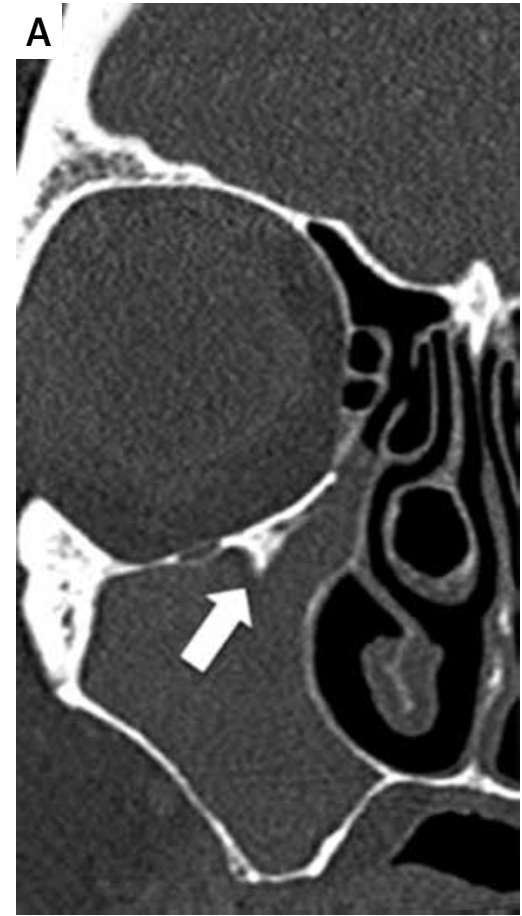

\section{Discussion}

The modified transnasal endoscopic medial maxillectomy (MTEMM) was described by Suzuki et al. in 2011 [7]. It provides wide access to the maxillary sinus including the prelacrimal recess and allows for removal of maxillary sinus tumors with preservation of the nasolacrimal duct and inferior nasal turbinate. Different variants of this procedure were presented in the literature in the following years.

Uniform nomenclature of these procedures is still not determined. However, it seems that the term "maxillectomy" is inappropriate since no part of the maxilla is removed.

"Prelacrimal recess approach" is currently the term most often used for this procedure. Recently Suzuki et al. presented results of treatment of 51 patients operated on for inverted papilloma using this method. The authors obtained good functional results with a low recurrence rate [3]. Piriform aperture enlargement performed to gain control over the anterior wall of the sinus in some of these procedures may cause collapse of the nasal valve and/or cosmetic deformity similarly as after the Denker approach. Zhou et al. observed this complication in $4 \%$ of 71 patients (mean observation of 37.3 months) [4].

Endoscopic postoperative follow up after the prelacrimal approach is possible only if wide MMA is performed. Visualization of the anterior part of the maxillary sinus is then possible only with the use of a fiberscope. This can be difficult due to the poor spatial orientation inside the sinus. Taking a biopsy under local anesthesia in these cases can also be problematic.

In $30 \%$ of patients the nasolacrimal duct is partially or completely fused with the anterior wall of the maxillary sinus, and the lacrimal recess is narrow or missing. In these cases the prelacrimal approach can be difficult to perform [8].

The IMA has been long known as an independent approach to the maxillary sinus or part of the Caldwell-Luc procedure. In the era of functional endoscopic sinus surgery it has been nearly abandoned, as it does not improve the natural drainage of the sinus. However, IMA offers good insight into the anterior part of the maxillary sinus [9] and provides an auxiliary aeration and drainage pathway [5, 10]. IMA does not affect natural ciliary transport from the maxillary sinus in presence of functioning natural ostium [11].

The combined antrostomies approach has been proven effective in treatment of a severely diseased maxillary sinus [10]. There were no cases of epiphora or floppy inferior turbinate in 34 patients treated with this method in 1-year observation.

This technique allowed us to use antrostomies as independent ports for the surgical tool and scope, 

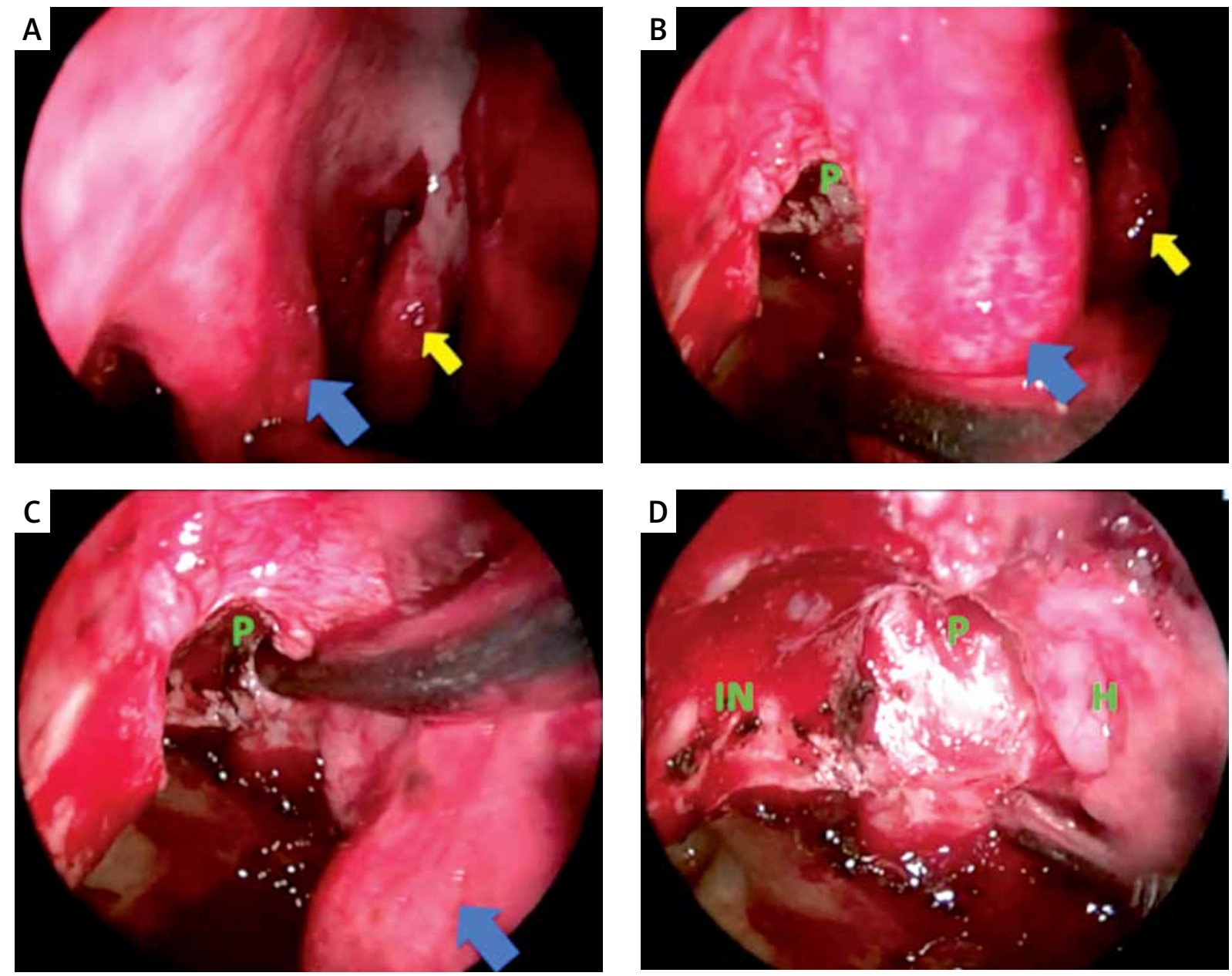

Photo 4. Intraoperative view of the right nasal cavity, maxillary sinus and prelacrimal recess $\left(45^{\circ}\right.$ scope, patient 5). A, B - right nasal cavity view: yellow arrow - middle turbinate, blue arrow - inferior nasal turbinate, $\mathrm{P}$ - prelacrimal recess, C, D - enlarged inferior antrostomy view on the prelacrimal recess: IN - infraorbital nerve, $\mathrm{H}$ - Hasner's valve

which improved visibility and maneuverability within the sinus. In cases with attachment on the anterior wall IMA was used to introduce both the endoscope and the surgical tool. In our hands adequate visualization of the lower anterior and entire lateral wall of the maxillary sinus could be achieved at a 70-90-degree viewing angle after enlarging the IMA anteriorly to the level of the pyriform aperture.

In contrast, visualization and the possibility of introducing surgical tools to the prelacrimal recess was unsatisfactory in some patients.

In one of our patients with prelacrimal recess involvement (Photos 4 and 5) we managed to visualize the prelacrimal recess and remove the IP completely through the enlarged IMA due to favorable anatomical conditions (wide prelacrimal recess, short nasolacrimal duct). In the second patient, with a relatively narrow prelacrimal recess, we decided to extend combined antrostomies to the prelacrimal approach because of inadequate visualization of the medial wall of the nasolacrimal canal. There are no data on late functional outcomes of combination of these two approaches (combined antrostomies + prelacrimal recess) in a larger group of patients. However, the junction between the lateral wall of the nasal cavity and the floor of the nasal cavity is fractured during the pre-lacrimal approach anyway, so addition of inferior antrostomy should not be considered more destructive.

Combined CT and MRI assessment provides more useful information than using one of these techniques alone and increases the chance of identifying the attachment (Photos 2 and 3) [6]. In our study 

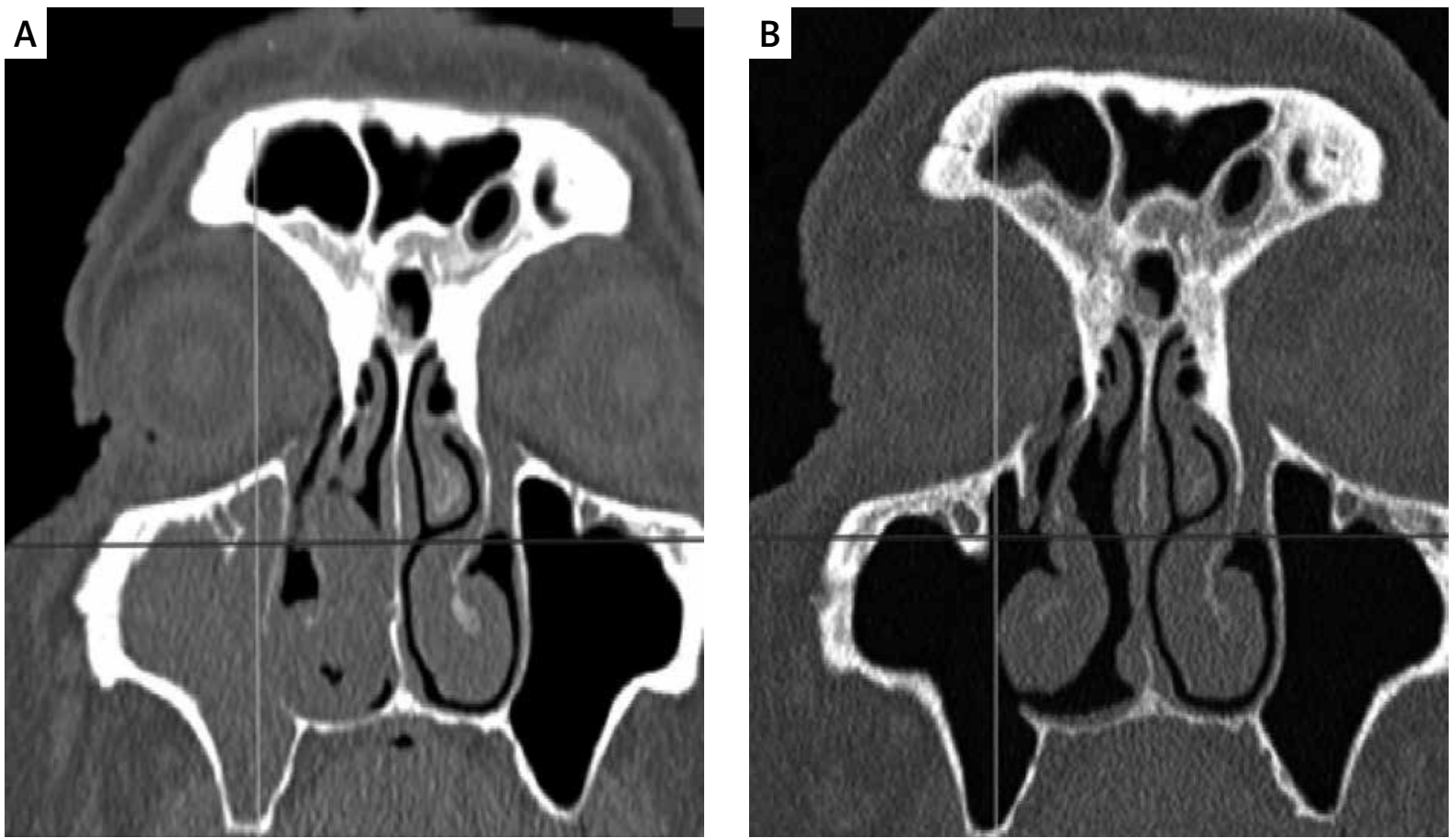

Photo 5. Patient with prelacrimal recess involvement from Photo 3 (patient 5): A - preoperative and B - postoperative coronal CT view

group we identified the attachment in 10 out of 14 patients preoperatively, although MR was available only in 4 of them. Thus involvement of the prelacrimal recess can be predicted in the majority of patients. In most primary IPs preoperative identification of the attachment allows for planned, patient-tailored endoscopic surgery (combined antrostomies or the prelacrimal approach). If the attachment is not visible on preoperative CT and MRI, we suggest starting the surgery with combined antrostomies. In case of inadequate prelacrimal recess control the next step should be the prelacrimal recess approach. Endoscopic middle maxillectomy should be used as the last option. There was no need for the transseptal approach, adjuvant Caldwell-Luc approach, or endoscopic middle maxillectomy in our patients. However, the small number of subjects is the major limitation of our study. In patients with involvement of the deep alveolar recess, multiple attachment sites or carpet-like growth over a large area, combined antrostomies would probably be insufficient.

\section{Conclusions}

The combined antrostomies approach is effective and safe in treatment of maxillary sinus inverted papilloma. It seems superior to medial maxillectomy, as it is associated with lower morbidity. It offers the possibility of easy endoscopic follow-up through inferior antrostomy in contrast to the prelacrimal approach. Preliminary data suggest that in case of pelacrimal recess involvement this approach can be safely extended to the prelacrimal approach.

\section{Conflict of interest}

The authors declare no conflict of interest.

\section{References}

1. Tong CCL, Patel NN, Maina IW, et al. Inverted papilloma with multifocal attachment is associated with increased recurrence. Int Forum Allergy Rhinol 2019; 9: 865-9.

2. Suh JD, Chiu AG. What are the surveillance recommendations following resection of sinonasal inverted papilloma? Laryngoscope 2014; 124: 1981-2.

3. Suzuki M, Nakamura Y, Yokota M, et al. Modified transnasal endoscopic medial maxillectomy through prelacrimal duct approach. Laryngoscope 2017; 127: 2205-9.

4. Zhou B, Huang Q, Sun J, et al. Resection of inverted papilloma of the maxillary sinus via a prelacrimal recess approach: a multicenter retrospective analysis of surgical efficacy. Am J Rhinol Allergy 2018; 32: 518-25. 
5. Moeller CW, Stankiewicz JA. Endoscopic inferior meatal antrostomy. Oper Tech Otolaryngol Head Neck Surg 2010; 21: 156-9.

6. Fang G, Lou H, Yu W, et al. Prediction of the originating site of sinonasal inverted papilloma by preoperative magnetic resonance imaging and computed tomography. Int Forum Allergy Rhinol 2016; 6: 1221-8.

7. Suzuki M, Nakamura Y, Nakayama M, et al. Modified transnasal endoscopic medial maxilectomy with medial shift of preserved inferior turbinate and nasolacrimal duct. Laryngoscope 2011; 121: 2399-401.

8. Sieśkiewicz A, Buczko K, Janica J, et al. Minimally invasive medial maxillectomy and the position of nasolacrimal duct: the CT study. Eur Arch Otolaryngol 2016; 274: 1515-9.

9. Zhao Y, Cheng J, Yang J, et al. Modified endoscopic inferior meatal fenestration with mucosal flap for maxillary sinus diseases. Videosurgery Miniinv 2018; 13: 533-8.

10. Albu S, Gocea A, Necula S. Simultaneous inferior and middle meatus antrostomies in the treatment of the severely diseased maxillary sinus. Am J Rhinol 2011; 25: 80-5.

11. Ochi K, Sugiura N, Komatsuzaki Y, et al. Patency of inferior meatal antrostomy. Auris Nasus Larynx 2003; 30 (suppl): S57-60.

Received: 23.10.2019, accepted: 8.12.2019. 